\title{
Proof-of-principle experiment for laser-driven acceleration of relativistic electrons in a semi-infinite vacuum
}

\author{
T. Plettner and R. L. Byer \\ E. L. Ginzton Laboratories, Stanford University, Stanford, California 94305, USA \\ E. Colby, B. Cowan, C. M. S. Sears, J.E. Spencer, and R. H. Siemann \\ Stanford Linear Accelerator Center, Menlo Park, California, 94025, USA \\ (Received 22 September 2005; published 13 December 2005)
}

\begin{abstract}
We recently achieved the first experimental observation of laser-driven particle acceleration of relativistic electrons from a single Gaussian near-infrared laser beam in a semi-infinite vacuum. This article presents an in-depth account of key aspects of the experiment. An analysis of the transverse and longitudinal forces acting on the electron beam is included. A comparison of the observed data to the acceleration viewed as an inverse transition radiation process is presented. This is followed by a detailed description of the components of the experiment and a discussion of future measurements.
\end{abstract}

DOI: 10.1103/PhysRevSTAB.8.121301

PACS numbers: 41.75.Jv, 41.75.Ht, 42.25.Bs

\section{INTRODUCTION}

Laser-driven particle acceleration in vacuum shows promise for substantially increased energy gradients for future advanced accelerators [1]. Soon after the appearance of $Q$-switched high peak power lasers methods for accelerating charged particles were first explored [2-4]. It was realized that certain laser beam configurations in vacuum produce an electric field component parallel to the direction of motion of the electron beam and thus create a linear acceleration force. However, due to the mismatch of the phase velocity of the electromagnetic wave and the particles no energy transfer to the electron beam through this linear acceleration mechanism can occur in free space [5]. This implies the necessity of an accelerator structure that limits the interaction of the laser field with the electrons or slows the effective phase velocity of the laser observed by the particles. The simplest such laser accelerator configuration, proposed by Pantell and Piestrup [6], is a single laser beam oriented at a shallow angle to the electron beam and terminated by a downstream boundary. This initial conceptual geometry was followed by the proposal of more refined arrangements employing crossed Gaussian beam configurations [7] and eventually conceptual staged-interaction laser accelerator structures that could in principle sustain very large continuous gradients [8-11].

We have carried out a proof-of-principle experiment using a single laser beam terminated by a single downstream boundary that led to the first experimental observation of laser-driven acceleration of relativistic electrons in a semi-infinite vacuum and confirmed the key physics for this particle acceleration process [12].

\section{THEORY}

The single-laser configuration employed for proof-ofprinciple experiment is illustrated in Fig. 1. A Gaussian laser beam is oriented at an angle $\alpha$ to the electron beam and terminated by a thin downstream boundary.

Calculation of the transverse and longitudinal force components. - The laser beam shown in Fig. 1 is polarized in the $x-z$ plane, oriented at an angle $a$ to the electron beam and focused at the boundary, assumed to be located at $z=$ 0 . The coordinates $\left(x^{\prime}, y^{\prime}, z^{\prime}\right)$ are aligned to the laser beam and rotated by an angle $a$ with respect to the coordinates $(x, y, z)$, which are aligned to the electron beam. The transverse electric field component of a TEM $\mathrm{T}_{00}$ Gaussian beam along its propagation axis $z^{\prime}$ in the slowly varying envelope approximation is [13]

$$
E_{\text {transverse }}\left(x^{\prime}, y^{\prime}, z^{\prime}, t\right)=\frac{E_{0} e^{-\left[x^{\prime 2}+y^{\prime 2} / w\left(z^{\prime}\right)^{2}\right]}}{\sqrt{1+z^{12} / z_{0}^{2}}} e^{i\left\{\omega t-k z^{\prime}-\eta\left(z^{\prime}\right)-\left[k x^{\prime 2} / 2 R\left(z^{\prime}\right)\right]-\varphi\right\}},
$$

where $E_{0}$ is the peak electric field amplitude of the laser beam, $\omega$ is the frequency of the laser, and $\lambda$ is the wavelength. $z_{0}$ is the Raleigh range given by $z_{0}=\pi w_{0}^{2} / \lambda$, $w\left(z^{\prime}\right)=w_{0}\left(1+\left(\lambda z^{\prime}\right)^{2} /\left(\pi w_{0}\right)^{2}\right)$ is the beam size located at $z^{\prime}$ where $w_{0}$ is the beam waist, $R\left(z^{\prime}\right)=z\left(1+z_{0}^{2} / z^{\prime 2}\right)$ is the radius of curvature at $z^{\prime}$, and $\eta\left(z^{\prime}\right)=\tan ^{-1}\left(z^{\prime} / z_{0}\right)$ is the Guoy phase shift for a $\operatorname{TEM}_{00}$ Gaussian laser beam at $z^{\prime} . \varphi$ is an arbitrary optical phase offset angle that determines whether the electron experiences acceleration or deceleration. Notice that $E_{\text {transverse }}$ is a scalar and to obtain the vector components the laser-crossing angle $\alpha$ and the laser polarization angle $\rho$ shown in Fig. 1 have to be taken into account. For a Gaussian beam there is a small longitudinal electric field component that can be approximated by 


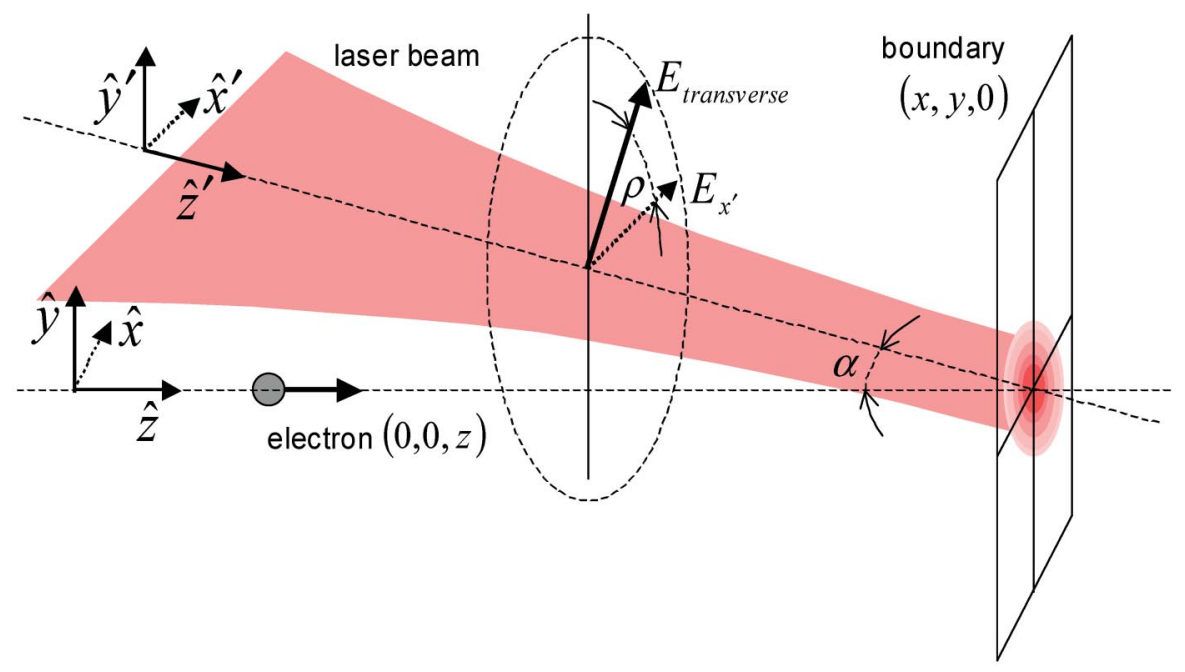

FIG. 1. (Color) Schematic diagram of the laser beam and the electron beam incident on a thin boundary.

applying Gauss's law $\nabla \cdot \vec{E}=0$ to the transverse electric field expression of Eq. (1) [14].

$$
E_{z^{\prime}}\left(x^{\prime}, z^{\prime}\right)=-2 x^{\prime}\left(\frac{1}{w\left(z^{\prime}\right)^{2}}+\frac{i k}{2 R\left(z^{\prime}\right)}\right) E_{x^{\prime}}\left(z^{\prime}\right)
$$

The electric field components in the $(x, z)$ coordinates aligned to the electron beam are found by the transformation of the coordinates $\left(x^{\prime}, z^{\prime}\right)$ in Eqs. (1) and (2) to the $(x, z)$ coordinates. The total longitudinal electric field along the $z$ axis is $E_{z}(z)=E_{z^{\prime}} \cos \alpha+E_{x^{\prime}} \sin \alpha$. The electron is assumed to follow the trajectory $z(t)=\beta c t$ where $\beta c$ is the velocity of the electron. Hence the energy gain of the electron from the semi-infinite interaction with this laser beam is the integral of the longitudinal electric field along the $z$ axis

$$
\Delta U=\int_{-\infty}^{0} q E_{z}(z) d z
$$

Figure 2 illustrates the energy gain of a relativistic electron interacting with a laser beam in the configuration shown in Fig. 1. In this particular example the optical phase offset of the laser $\varphi$ is chosen such that the energy gain is positive.
The importance of the boundary becomes clear from Fig. 2. In agreement with the Lawson-Woodward theorem [5] there is no energy gain of the electron if the boundary is absent.

For a Gaussian beam with a beam waist parameter $w_{0} \gg$ $\lambda$ and a crossing angle $|\alpha| \ll 1$ an analytic expression for the energy gain that approximates the laser beam with a plane wave can be derived. In this approximation the maximum possible energy gain in the semi-infinite laserelectron interaction scheme is equivalent to the path integral over half a slippage distance. Figure 2 illustrates the energy gain of a relativistic electron interacting with a laser beam in the configuration shown in Fig. 1. In this particular example the optical phase offset of the laser $\varphi$ is chosen such that the energy gain is positive.

$$
\Delta U \sim \frac{\lambda e E_{0}}{\pi} \frac{\alpha}{\frac{1}{\gamma^{2}}+\alpha^{2}} \cos \rho \cdot \cos \varphi
$$

$e$ is the electric charge, $\gamma$ is the time dilatation constant, and the other symbols have the same meaning as in Eq. (1). With this expression the energy gain follows the dependences $\Delta U \propto E_{0}, \quad \Delta U \propto \cos \rho, \Delta U \propto \lambda$, and $\Delta U \propto$ $\alpha /\left(1 / \gamma^{2}+\alpha^{2}\right)$ which has a maximum at a crossing angle $\alpha_{\max }=1 / \gamma$. For the parameters used in the experiment the

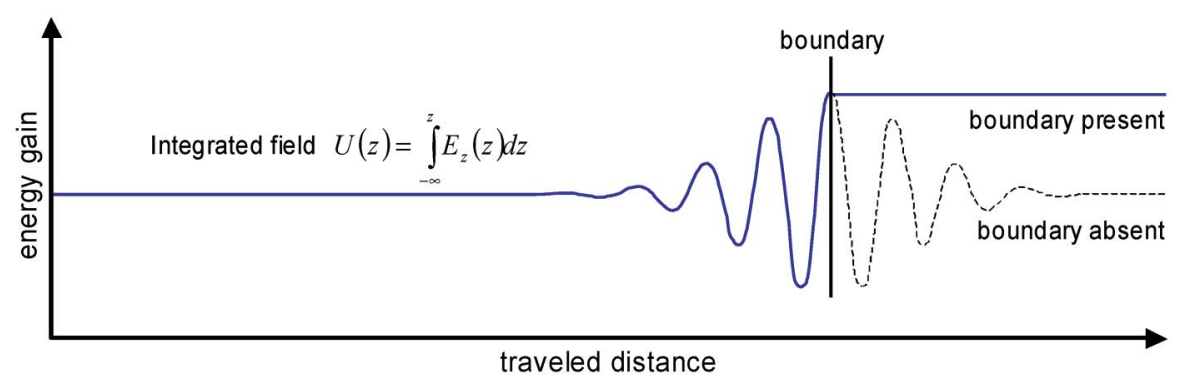

FIG. 2. (Color) Integrated field seen by a traveling electron along the $z$ axis. 


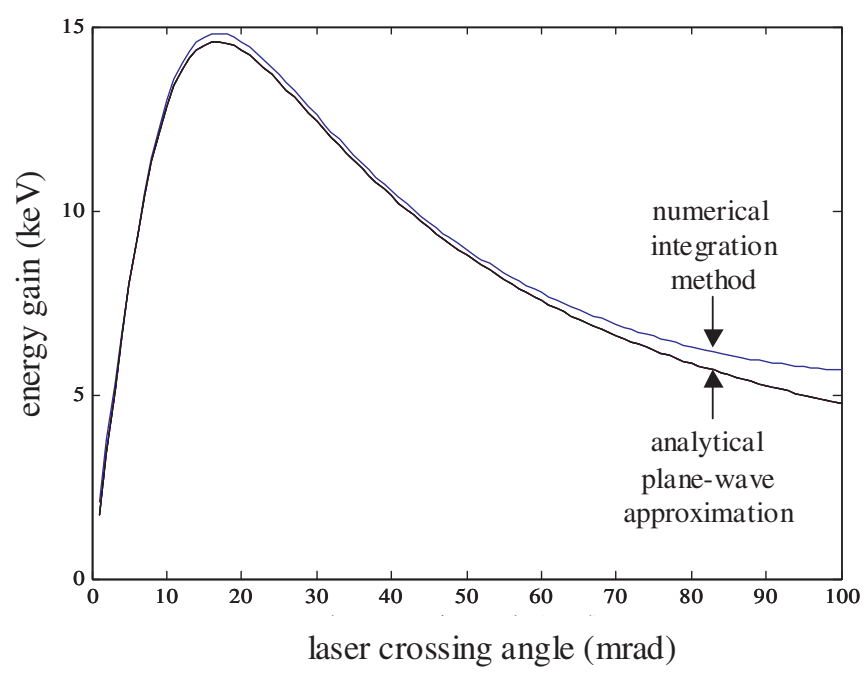

FIG. 3. (Color) Expected electron beam energy gain versus laser-crossing angle with $\gamma=60, E_{0}=2 \mathrm{GV} / \mathrm{m}$.

plane-wave solution in Eq. (4) is a very good approximation to the expected energy gain from numerical integration of the longitudinal electric field.

The lateral deflection forces. - The use of one laser beam implies residual transverse electric and magnetic fields components and hence it is important to estimate the possible effect of the lateral deflection forces and compare it to the longitudinal force. The force acting on the electron is $\vec{F}=q(\vec{E}+\vec{v} \times \vec{B})$. For a laser polarized horizontally (with the electric field in the $x$ - $z$ plane, $\rho=0$ ) $\vec{E}=E \cos \alpha \hat{x}+E \sin \alpha \hat{z}, \vec{B}=E / c \hat{y}$. The electron trajectory is along the $z$ axis $\vec{v}=\beta c \hat{z}$ and hence the resulting force components are

$$
F_{x}=q E(\cos \alpha-\beta), \quad F_{y}=0, \quad F_{z}=q E \sin \alpha .
$$

With $\gamma \gg 1$ and at a crossing angle $\alpha \sim \alpha_{\max }=1 / \gamma$ the transverse force in Eq. (5) becomes zero. For other typical values of $\alpha$ employed in the experiment the ratio of the transverse to longitudinal force is $\left|F_{x}\right| /\left|F_{z}\right| \leq 10^{-3}$. A similar type of calculation assuming the laser beam polarized in the $\hat{y}$ axis yields a force

$$
F_{x}=0, \quad F_{y}=q E(1-\beta \cos \alpha), \quad F_{z}=0 .
$$

Equation (6) shows that there is a residual vertical deflection force when the laser is vertically polarized. However with the typical experimental parameters this deflection force $F_{y}$ is only about $1 / 60$ compared to the acceleration force $F_{z}$ when the laser is horizontally polarized. In addition, the laser-driven particle acceleration effect was detected by a 1:1 imaging energy spectrometer with the laser-electron interaction occurring at the image plane and a scintillator screen located at the focal plane of the spectrometer. This arrangement is unsuitable to detect possible lateral deflections occurring as a result of the interaction with the laser. Hence the residual lateral forces do not interfere with the observed energy gain and for the purpose of this experiment can be neglected.

Comparison with the inverse-radiation picture. - Laserdriven particle acceleration has also been regarded as an inverse-radiation process $[15,16]$, which is equivalent to the path integral method displayed in Eq. (4), and in essence is a direct consequence of Poynting's theorem [17]. In the inverse-radiation picture the energy gain of the particle is the overlap integral of the laser field and the radiation field caused by the structure or medium.

$$
\Delta U=-\int_{-\infty}^{\infty} \oint_{S} \frac{2}{Z_{0}}\left(\vec{E}_{\text {laser }} \cdot \vec{E}_{\text {rad }}\right) d s d t .
$$

It is evident that Eq. (7) obeys the Lawson-Woodward theorem since there is no $\vec{E}_{\text {rad }}$ in free space. For the specific case of an infinite conducting boundary $\vec{E}_{\text {rad }}$ is the wellknown transition radiation cone having a radial polarization. Applying the transition radiation far-field electric field for $\vec{E}_{\text {rad }}$, the laser Gaussian beam for $\vec{E}_{\text {laser }}$, and making the same assumptions as for derivation of the analytical energy gain expression given in Eq. (4) the overlap integral in Eq. (7) becomes [18]

$$
\Delta U \sim \frac{\lambda e E_{0}}{\pi} \frac{\alpha}{\frac{1}{\gamma^{2}}+\alpha^{2}} \cos \rho \cdot \cos \varphi,
$$

which is identical to Eq. (4). This can be interpreted as follows: The Gaussian laser beam intersects a small sector of the radiation cone over which its polarization is nearly uniform and therefore $\vec{E}_{\text {laser }} \cdot \vec{E}_{\text {rad }} \propto \cos \rho$. Furthermore, the overlap of $\vec{E}_{\text {laser }}$ and $\vec{E}_{\text {rad }}$ is optimized at the $1 / \gamma$ radiation cone angle. Finally, the laser field amplitude $E_{0}$ can be pulled out from the overlap integral and hence a linear dependence with the laser electric field is expected too.

Expected energy modulation observations. - The electron beam utilized for the experiment was not optically bunched. The electrons were evenly spread over all optical phase angles $\varphi$ described in Eq. (1). Therefore the laserdriven particle acceleration manifested itself as a modulation of the energy of the electron bunch instead of a net energy shift.

Figure 4(a) shows 10 real laser-off energy profiles observed at the spectrometer and the corresponding simulated laser-on energy profiles obtained by a simulated energy modulation of these initial laser-off profiles at the condition of optimum spatial and temporal overlap. The observed broadening corresponds to an energy modulation of $25 \mathrm{keV}$. The energy spectrum of a particular event was characterized by the FWHM of the observed energy spread. Figure 4(b) shows a simulation of the FWHM energy spread of the electron beam as a function of relative laser timing. The difference of the FWHM energy spread between the laser-on and the laser-off data at the condition of best temporal overlap is a measure of the maximum 
a) simulated energy profiles

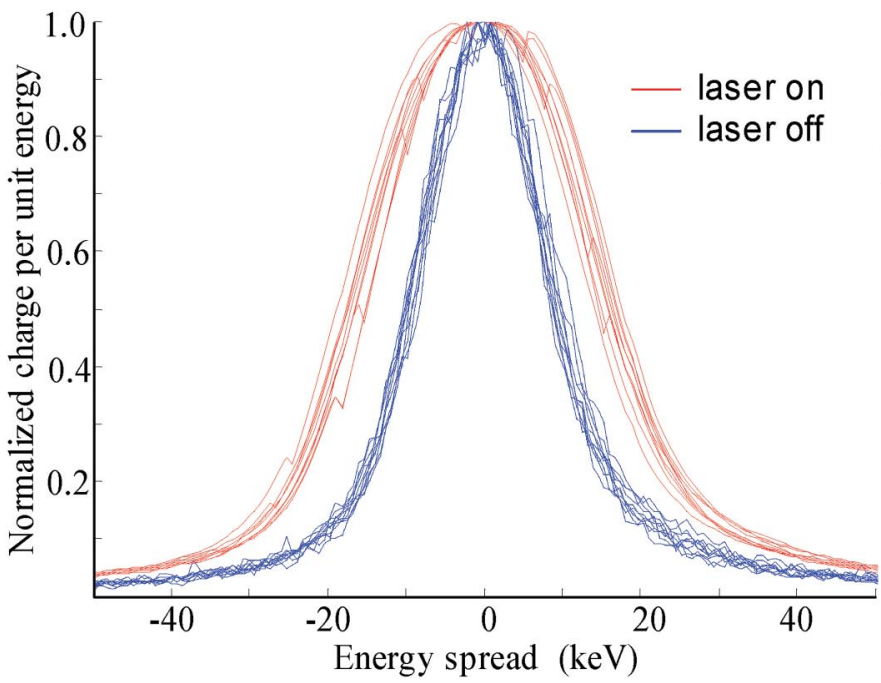

b) simulated laser time scan

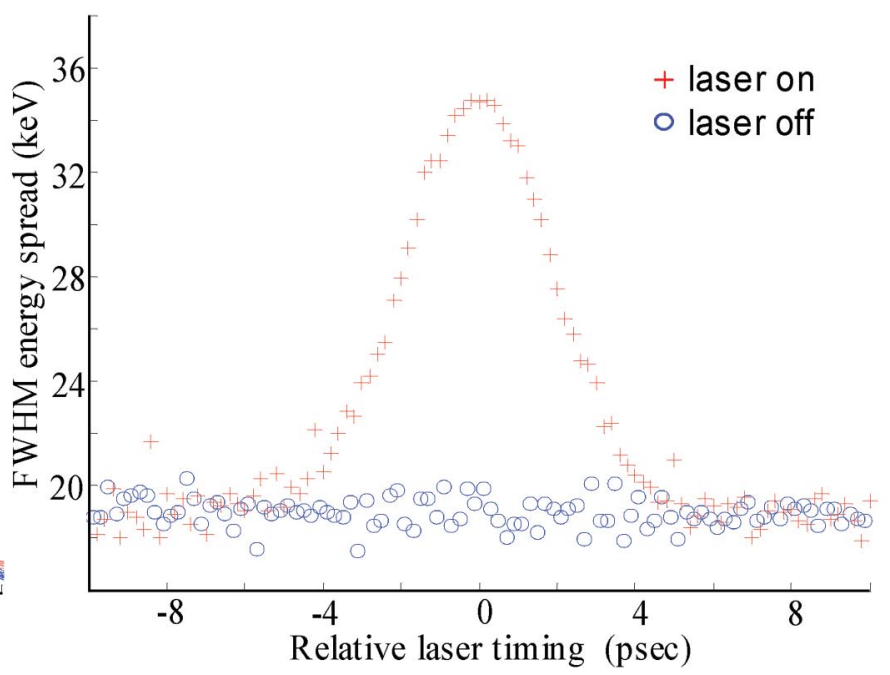

FIG. 4. (Color) Simulations using real laser-off electron beam energy profiles. The simulation employs an $\lambda=800 \mathrm{~nm}, T_{\mathrm{FWHM}}=$ 4 psec, $U_{\text {pulse }}=0.5 \mathrm{~mJ}, 100 \mu \mathrm{m}$ FWHM laser spot size and 16 mrad laser-crossing angle, and assumes a $30 \mathrm{MeV}, 2 \mathrm{psec}, 50 \mu \mathrm{m}$ wide electron bunch. (a) Laser-off and simulated laser-on energy profiles at the condition of optimum temporal overlap. (b) Simulated laser time scan using the laser-off data from the same run. Every second laser-off profile is used for the energy modulation simulation and its resulting energy spread is shown as laser-on data.

energy modulation under the given laser and electron beam conditions.

\section{THE EXPERIMENT}

The source of relativistic electrons for proof-of-principle experiment was the SCA-FEL facility at Stanford University. The primary choice for carrying out our experiment at this facility is the low energy spread of the $30 \mathrm{MeV}$ electron beam from the superconducting accelerator. Table I summarizes the important laser and electron beam parameters.

TABLE I. Laser and electron beam parameters of the LEAP experiment.

\begin{tabular}{lc}
\hline \hline \multicolumn{2}{c}{ Laser beam parameters } \\
Wavelength $\lambda$ & $800 \mathrm{~nm}$ \\
Waist FWHM spot size & $110 \mu \mathrm{m}$ \\
FWHM pulse duration & $2-4 \mathrm{psec}$ \\
Crossing angle $\alpha$ & $3-20 \mathrm{mrad}$ \\
Laser pulse energy & $\frac{1}{2} \mathrm{~mJ} / \mathrm{pulse}$ \\
Laser repetition rate & $1 \mathrm{kHz}$ \\
\multicolumn{1}{c}{$\quad$ Electron beam parameters } & \\
Beam energy & $30 \mathrm{MeV}$ \\
Macro pulse repetition rate & $10 \mathrm{~Hz}(\mathrm{typical})$ \\
Micro pulse repetition rate & $11.7 \mathrm{MHz}$ \\
FWHM spot size at the focus & $50 \mu \mathrm{m}$ \\
FWHM pulse duration & $1-2 \mathrm{psec}$ \\
Initial energy spread & $25-30 \mathrm{keV}$ \\
Charge/bunch at the experiment & $\sim 10 \mathrm{pC}$ \\
\hline \hline
\end{tabular}

The electron beam line elements. - The SCA-FEL facility employs six $L$-band superconducting accelerator segments that produce a $\sim 1$ msec long electron beam pulse train (macro pulse) with an electron bunch spacing of $85 \mathrm{nsec}$. Two free-electron lasers (FELs) are installed on this beam line but are detuned so they do not lase during the laser-acceleration experiments. An energy collimator with an adjustable slit was installed at the exit chicane of the downstream FEL. This element was critical in reducing the energy spread jitter to produce a fairly consistent FWHM energy spread $<30 \mathrm{keV}$ for most events, allowing for the observation of a few-keV laser-driven energy modulation. Furthermore tuning of the rf phase in the last accelerator structure allowed for a time-energy correlation of the electrons in the bunch. With such electron bunches the energy collimator also served as a coarse filter for timing jitter.

Because of the repetition rate of the laser only one laser pulse could be overlapped with an electron bunch within the macro pulse. To reject the remaining $\sim 10^{4}$ electron bunches a fast kicker was placed downstream of the energy collimator, directing the selected single electron bunch into a separate electron beam line. This was an important element in eliminating the background of noninteracting electron pulses that would have buried the laseracceleration signature on the spectrometer and also helped reduce $g$ ray damage of sensitive equipment located near the interaction region. The angular deflection delivered by the kicker was on the order of a few mrad. A sextupole magnet located $\sim 1 \mathrm{~m}$ further downstream amplified the deflection angle to $5^{\circ}$. The electron bunch was transported 
with an approximate beam diameter $2-3 \mathrm{~cm}$ before reaching a focusing triplet located $1 \mathrm{~m}$ upstream of the laserelectron interaction region where it was reduced to a $\sim 50 \mu \mathrm{m}$ spot. Downstream of the interaction region with the laser the electron beam entered a $90^{\circ}$ bending magnet and was projected to a Ce:YAG scintillator screen at the focal plane of the magnet. Figure 5 illustrates the electron beam transport.

The laser system.-We employed a Ti:sapphire based regenerative amplified psec laser system available at the SCA-FEL facility. The system consists of a 1 Watt, $\lambda=$ $800 \mathrm{~nm}, f_{\text {rep }}=80 \mathrm{MHz}$ mode locked oscillator followed by a regenerative amplifier delivering a maximum laser pulse energy of $1 \mathrm{~mJ}$. Adjustments in the pulse compressor at the exit of the regenerative amplifier allowed for laser pulse durations between 2 and 15 psec FWHM. The repetition rate of the regenerative amplifier was matched to the macro pulse repetition rate of the electron beam, which was typically set at $10 \mathrm{~Hz}$. The mode locked laser was rflocked to the electron beam. The trigger timing of the regenerative amplifier, which controlled the amplified laser pulse timing in increments of the oscillator period was used to set the coarse timing of the laser pulse arriving at the experiment. The $\sim \frac{1}{2} \mathrm{nsec}$ scale time adjustments were made by selection of cable lengths into the mode locked laser rf phase-lock loop circuit, and the fine scale psec time adjustments were achieved by a voltage controlled phase shifter in front of the phase-lock loop circuit. Because of the location of the laser room at the SCA-FEL facility a $\sim 100 \mathrm{~m}$ evacuated laser transport line was required to deliver the laser pulse to the experiment. Thermal expansion and vibration present in the building made active laser position feedback necessary.

The interaction chamber components. - The key elements of the experiment consisted of a vacuum interaction chamber containing the accelerator cell and spatial and temporal beam overlap diagnostics [19] followed by the high-resolution energy spectrometer. The electron and laser beams were aligned with a set of removable Ce:YAG screens placed at various locations along the beam trajectory and monitored by long distance microscopes. To ensure spatial overlap with the electron beam the laser beam FWHM spot size was set to $110 \mu \mathrm{m}$. The laserelectron crossing angle was adjustable between 3 and $20 \mathrm{mrad}$. A streak camera was employed to determine the coarse relative timing between the laser and the electron beam. To ensure temporal overlap with the electron beam the laser pulse duration was set to $2-4$ psec.

An inverse free-electron laser (IFEL) located $10 \mathrm{~cm}$ upstream of the tape boundary served as a high-resolution absolute timing monitor for the laser and the electron beam. As shown in Fig. 6 the spatial beam alignment requirements for the IFEL and the vacuum laser acceleration were chosen to be incompatible to prevent interference of the IFEL with the laser acceleration and to allow for a series of separate experiments involving the IFEL [20].

The IFEL had more relaxed beam alignment conditions and showed a very strong energy modulation, making it a useful laser-timing tool that is easy to implement. As a first step the laser and the electron beam were aligned and focused for the IFEL operation as shown in Fig. 6(a). Once the laser timing for IFEL interaction had been found the laser and electron beams were realigned for the vacuum laser-driven particle acceleration as shown in Fig. 6(b).

The optical boundary. - The optical boundary that limits the laser interaction with the electron beam is a $8 \mu \mathrm{m}$ thick, $230 \mathrm{~m}$ long kapton tape with a $1 \mu \mathrm{m}$ thick reflective gold coating that was moved to a new location for each laser shot. This moving-tape arrangement enabled us to make the boundary "disposable" and operate the laser above the damage threshold fluence of the tape surface. This approach circumvented the damage threshold limitation encountered in previous dielectric permanent accelerator cell approaches [21] and allowed us to operate the laser at full power and to maximize the signal to noise in the experiment. The laser pulse energy available at the tape boundary was $\frac{1}{2} \mathrm{~mJ}$ per pulse. Our design essentially amounts to a modified "Walkman"-type audio tape player

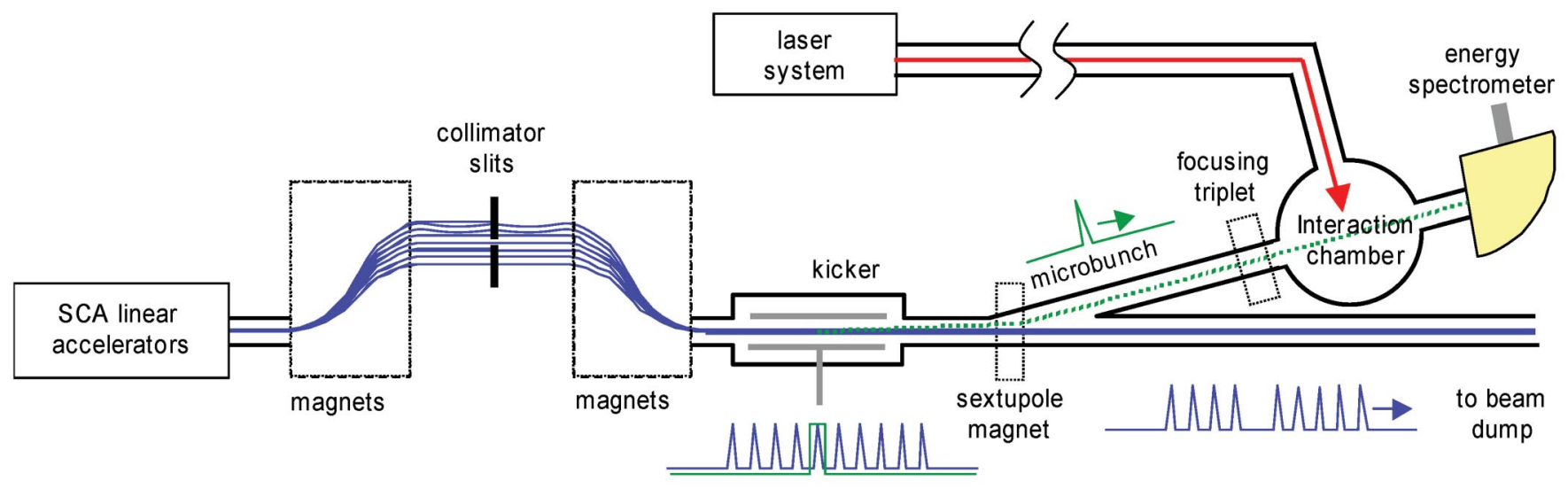

FIG. 5. (Color) Diagram of the electron beam line at the SCA-FEL facility. 


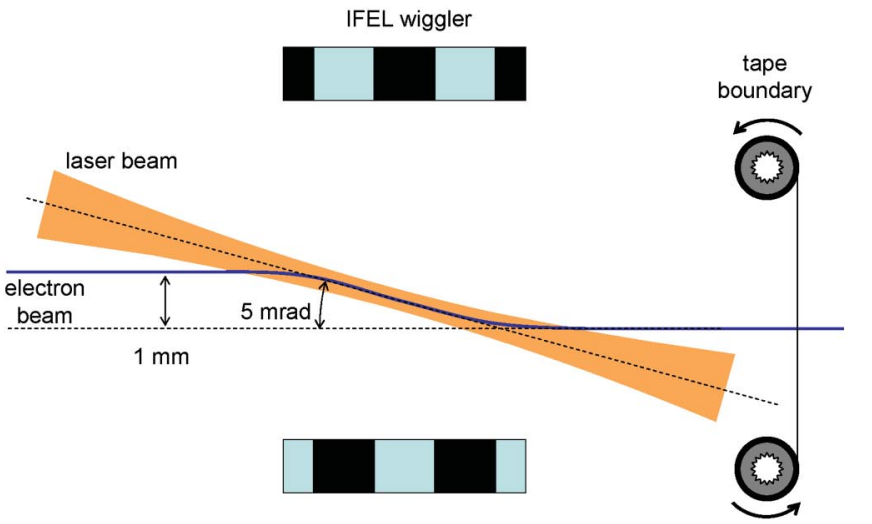

(a)

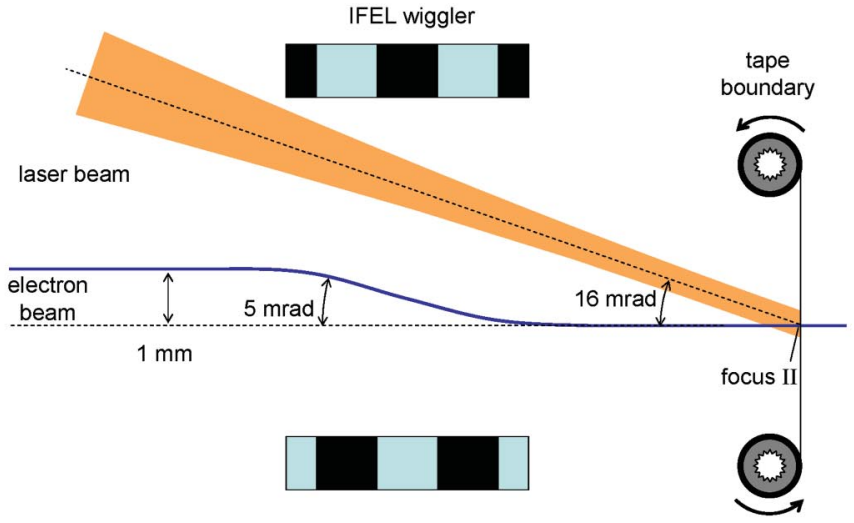

(b)

FIG. 6. (Color) (a) laser beam alignment configuration for the IFEL operation and (b) the tape boundary operation. The location of the laser beam focus was adjusted by a sliding telescope. The transverse dimensions in the figures are exaggerated. The IFEL gap was $2 \mathrm{~mm}$ and the distance of the center of the IFEL gap to the tape boundary was $10 \mathrm{~cm}$.

placed in vacuum. Figure 7(a) shows a photograph of the movable tape drive.

An important aspect of laser ablation dynamics for ultrashort laser pulses is that during the first few picoseconds the material forms an overdense plasma where the ionized atoms have not moved significantly. This keeps the surface a highly reflective metal-like boundary during that phase of the ablation process. A series of separate experiments (see Appendix A) were carried out before implementing the tape and verified the expected laser ablation dynamics process $[22,23]$, confirming the presence of the reflective gold boundary during the ablating laser pulse, provided its duration is 10 psec or shorter. We typically operated at laser pulse durations of 2-4 psec for our laser-acceleration experiments. Hence, in spite of operating above the laser damage threshold of the gold surface the tape remained an effective terminating boundary for the laser and the electron beam traversed the vacuum space and boundary prior to ejection of material. Finally, employing a very thin boundary of $8 \mu \mathrm{m}$ thickness allowed for running the electron beam through the tape and avoid the tight electron beam alignment requirements for transmission through few-micron wide aperture slits. Appendix B presents a calculation of the expected energy

\section{a) the tape drive unit}

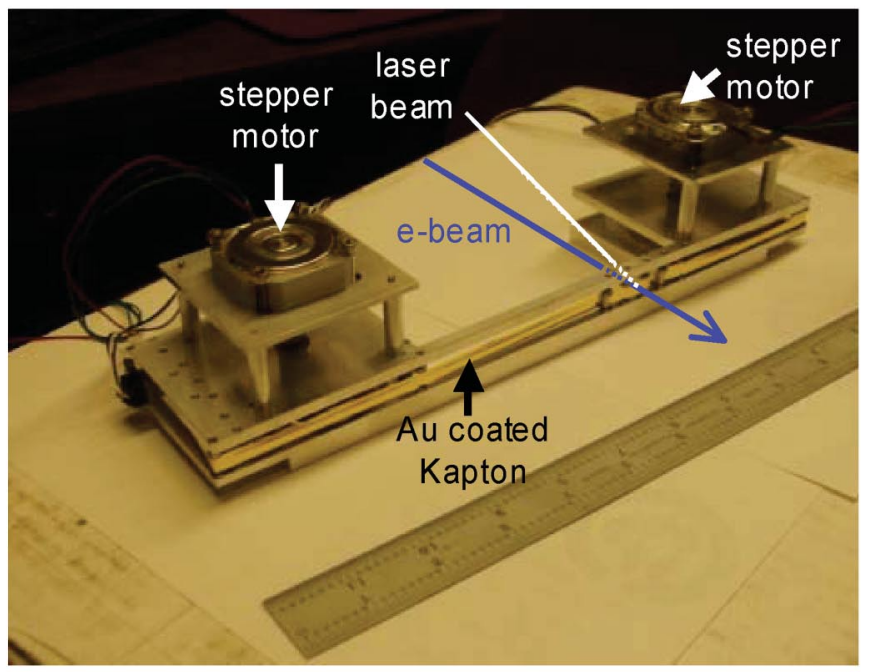

\section{b) the interaction chamber components}

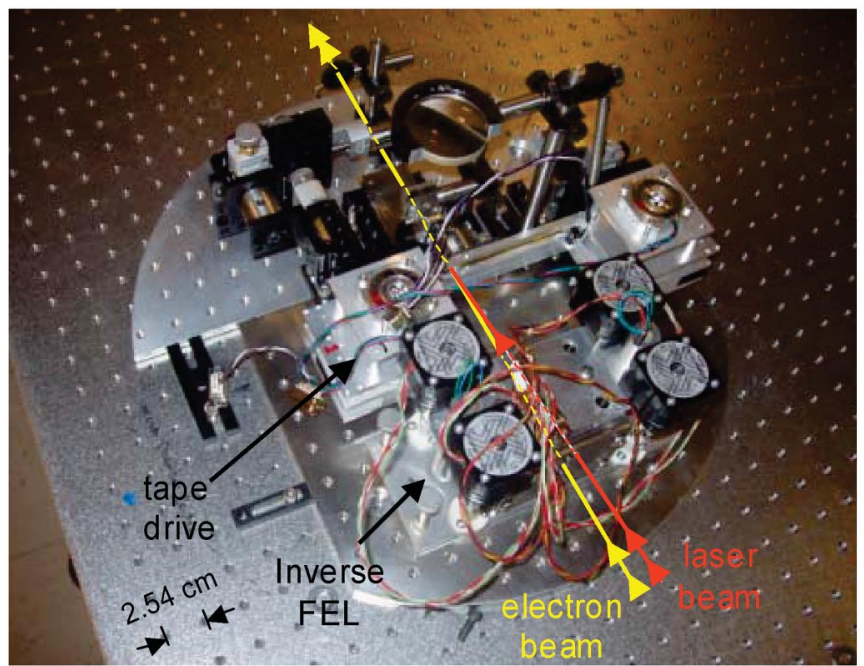

FIG. 7. (Color) (a) The tape drive for the disposable Au-coated Kapton boundary. A pair of stepper motors is used to wind the tape to either direction and a pair of opto-switches detects the end of tape. (b) The breadboard containing the vacuum chamber components, showing the IFEL and the tape drive unit located downstream. 
broadening effect caused by the foil. As a result of these simplifications the beam tuning time decreased substantially and efforts could be concentrated on data collection, ultimately leading to the observation laser-driven particle acceleration. Figure 7(b) shows the entire interaction region assembly including the IFEL, the tape boundary and diagnostics on a breadboard prior to being placed inside the vacuum chamber.

The energy spectrometer. - A high-resolution energy spectrometer located downstream of the tape drive was used to measure the energy spread of the electron beam and to quantify the increase of energy spread resulting from energy modulation of the electron beam caused by the laser. The interaction region and the image plane for the spectrometer were chosen to be equidistant such that energy spectrometer becomes a 1:1 imaging system in the horizontal coordinate. This configuration eliminated any possible interference of later deflections caused by the laser to appear as an acceleration signature. The spectrometer was a dc current, $\frac{1}{2} \mathrm{~m}$ radius, $90^{\circ}$ bending magnet with a resolving power of $P / \Delta P>10^{4}$, corresponding to an energy resolution of about $2 \mathrm{keV}$. A $2.5 \mathrm{~cm}$ diameter, $\frac{1}{2} \mathrm{~mm}$ thick YAG fluorescent screen located at the focal plane of the spectrometer monitored by a gated image intensified 16-bit camera. With a scan of the magnet current for a fixed electron beam energy a camera pixel was determined to correspond to $0.7 \mathrm{keV}$ of energy. The data acquisition program labeled the image of each electron beam shot at the spectrometer with the corresponding experimental conditions for the particular image and stored it in a file containing the image sequence for the laser time scan in progress. External software was employed to ana- lyze the images and extract from these image-sequence files the energy profiles such as those shown in Fig. 8(a).

\section{MEASUREMENTS}

Measuring the energy modulation.-The laser-induced energy modulation of the electron beam manifested itself as a broadening of the energy spread of the electron beam. Figure 8(a) displays the observed energy spectra of the electron beam at the condition of spatial and temporal overlap with the laser. The blue traces correspond to the natural energy spread of the electron beam when the laser was not applied and the red traces correspond to the increased energy width of the electron beam when the laser was present. Because of timing jitter the data were collected in the form of laser time scans, where a series of $\sim 1000$ shots with a laser timing that was varied over a $\sim 20-30$ psec window were taken. To reveal the natural energy spread of the electron beam the laser was toggled on and off randomly. Figure 8(b) illustrates a typical laser time scan. Each laser time scan allowed us to find the maximum energy modulation as a function of the experimental parameters that we kept fixed during the scan. The experimental data shown in Fig. 8 are in good agreement with the energy modulation simulations of the laser-off data of the same run shown in Fig. 4. However, Fig. 4 neglects shot-to-shot jitter and hence shows no fluctuations in the laser time scan and energy broadening.

Simulations employing laser-off data showed that the change in the FWHM energy spread does not scale linearly with the applied modulation but instead scales in quadrature with energy modulation. The quadrature energy broadening for a particular laser-on energy profile is defined as a)

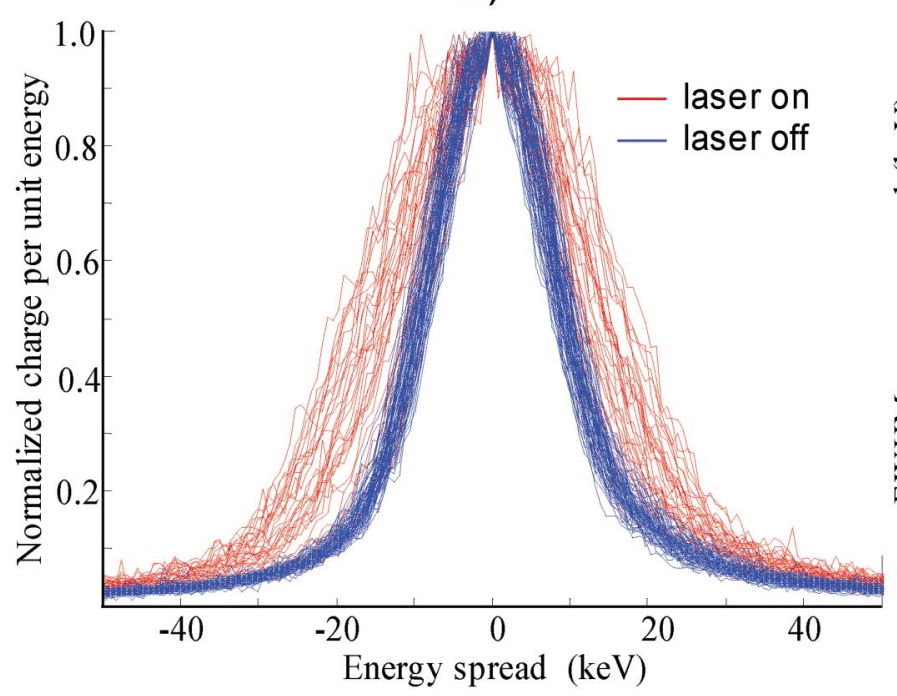

b)

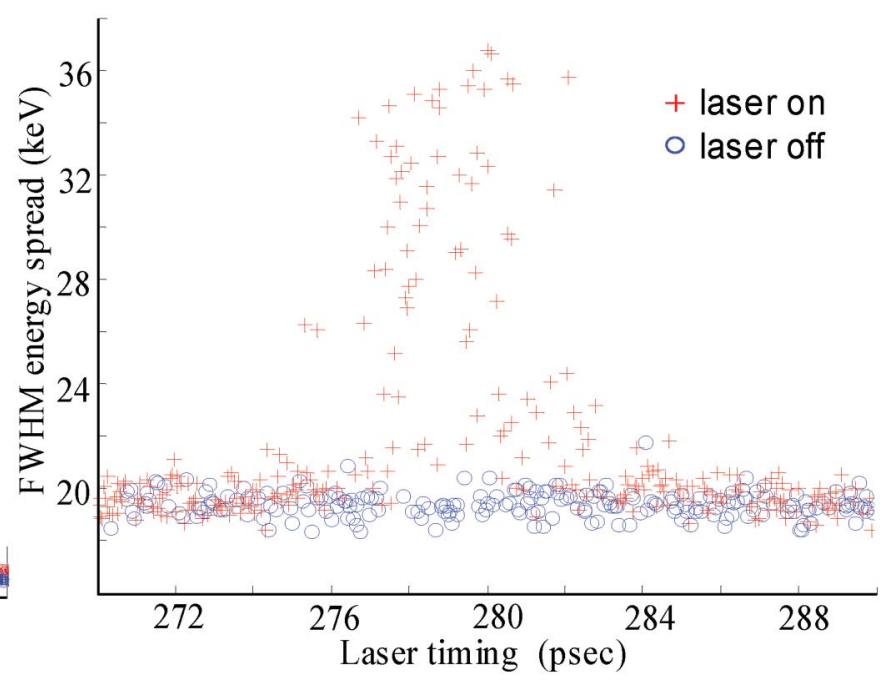

FIG. 8. (Color) (a) Experimentally observed laser-driven energy modulation near the condition of optimum temporal overlap. (b) Laser time scan showing the FWHM energy spread of the electron beam. 


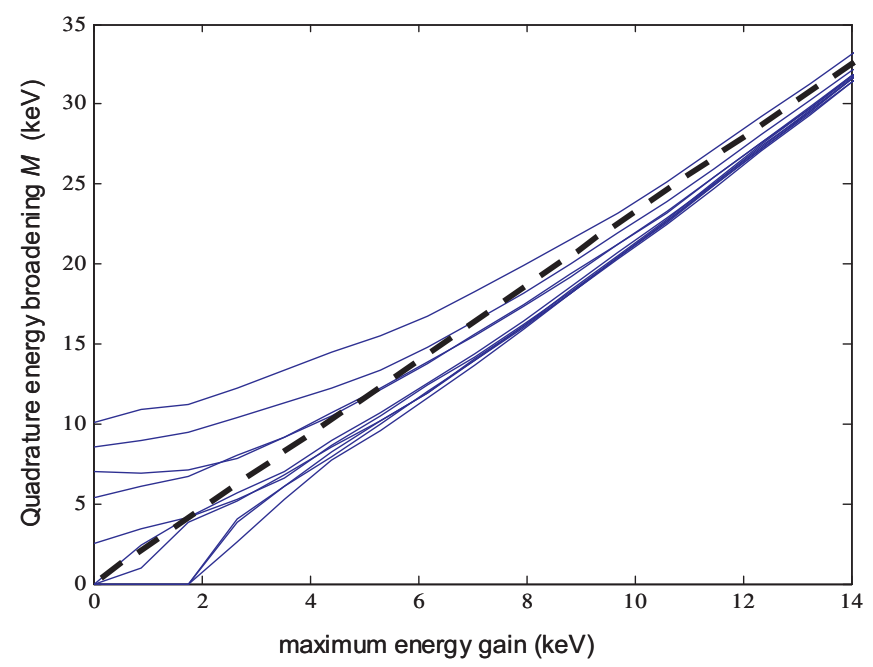

FIG. 9. (Color) Quadrature energy broadening $M$ as a function of maximum energy gain. The solid traces correspond to calculated values of $M$ from individual electron beam energy profiles and the dashed line is a linear fit of the data through the origin.

$$
M_{i}=\sqrt{F_{i}^{2}-\bar{F}_{\text {off }}^{2}}
$$

where $F_{i}$ is the FWHM energy spread of the $i$ th laser-on event, $\bar{F}_{\text {off }}$ is the average FWHM energy spread of the laser-off events, and $M_{i}$ is the quadrature energy broadening. Figure 9 shows the dependence of the quadrature energy broadening for 10 laser-off energy spectra as a function of the simulated energy gain, which corresponds to $\frac{1}{2}$ the modulation strength. As shown by the fit in Fig. 9, under optimum spatial and temporal overlap conditions the maximum quadrature energy broadening scales with the maximum energy gain as $M_{\max }=2.2 \Delta U_{\max }$. The simulations also reveal that for a given modulation strength $M$ shows a slight dependence on the initial electron beam energy profile, which as seen in Fig. 8(a) is nearly Gaussian. Finally, Fig. 9 shows that the quadrature energy broadening parameter $M$ cannot distinguish between energy gain values $<2 \mathrm{keV}$ and natural shot-to-shot fluctuations of the energy profiles.

The effect of the shot-to-shot jitter brings about a lowering of the average observed energy modulation value $\langle M\rangle$ around the condition of optimum time overlap. The average energy modulation $\langle M\rangle$ used in the data analysis contained $\sim 50$ events closest to the optimum laser timing. In Fig. 8(b) this corresponds to a 2 psec time window centered at 278 psec. With the help of simulations that include shotto-shot timing jitter observed at the SCA facility it was determined that the average energy modulation is close to $\frac{1}{2}$ the maximum energy broadening for the given conditions, $\langle M\rangle=0.45 M_{\max }$.

The laser-driven energy modulation was measured as a function of

(i) The presence of a field-terminating boundary

(ii) The laser electric field amplitude

(iii) The laser polarization

(iv) The laser-crossing angle.

The dependence of a field-terminating boundary.-To verify the Lawson-Woodward theorem two successive laser time scans, one with the boundary in place and the following with the tape moved out were taken. Figure 10 shows two successive laser time scans; Fig. 10(a) corresponds to data taken with the boundary in place, and Fig. 10(b) with the boundary removed. As observed in Fig. 10(a) there is a clear laser-driven energy modulation that peaks at a laser timing of 295 psec. In Fig. 10(b) it can a)

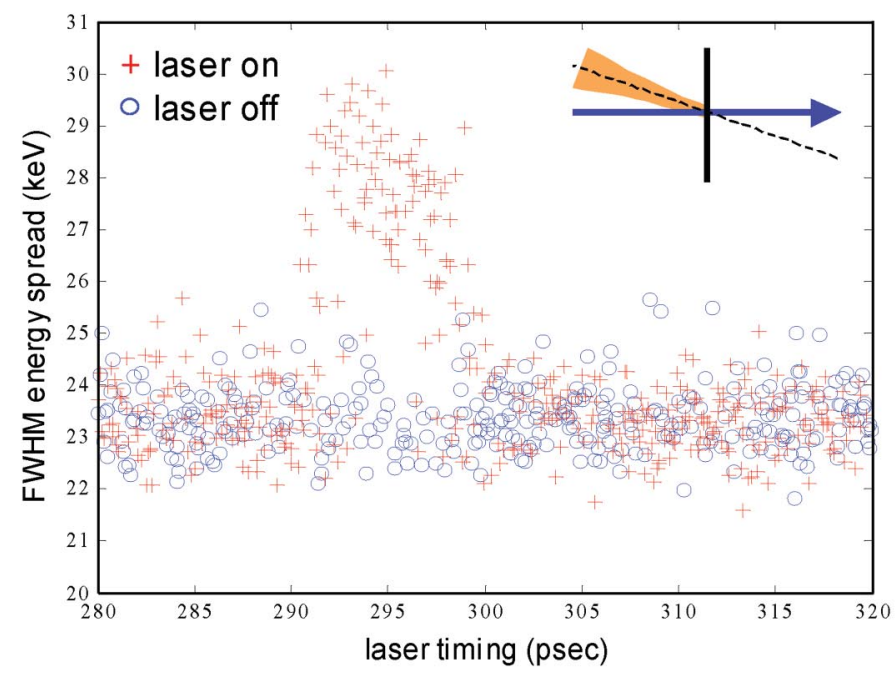

b)

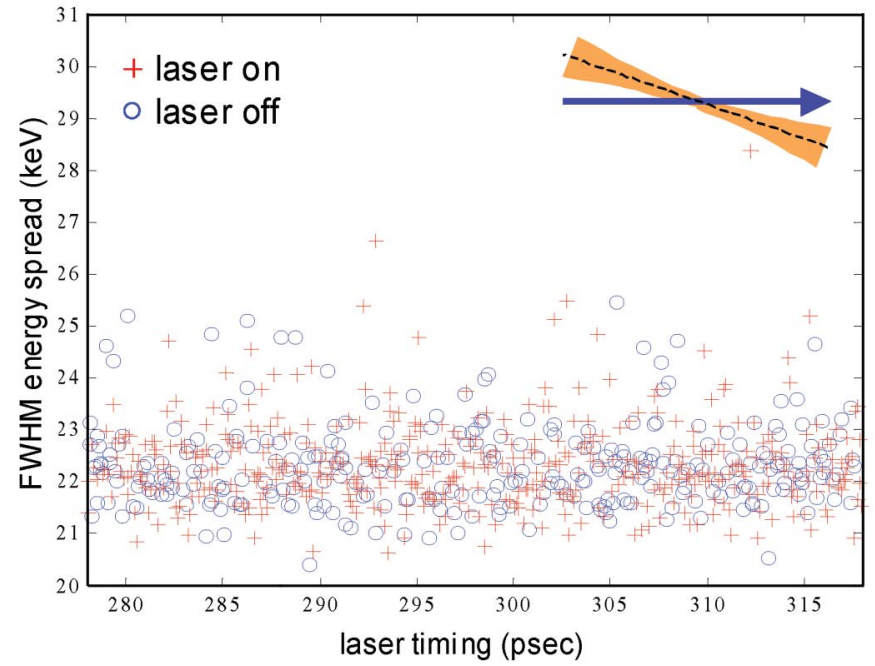

FIG. 10. (Color) The effect of the field-terminating boundary. 
be observed that the laser-induced energy modulation disappears when the boundary is removed. The laser-on and laser-off energy spread data in Fig. 10(b) were confirmed to be statistically identical with the statistical $t$ test [24].

The effect of the $8 \mu \mathrm{m}$ gold-coated Kapton film on the natural energy spread of the beam can be observed by comparing the baseline energy spread of Figs. 10(a) and 10 (b). There is a $1 \mathrm{keV}$ broadening of the energy spread of the electron beam as a result of the electrons traversing the tape (see Appendix B). As can be appreciated in Fig. 10(a) it is a small effect compared to the observed laser-induced energy broadening. Furthermore collection of the laser-off data during each laser time scan in the presence of the tape (under the same conditions as the laser-on data) allows us to isolate and measure the energy broadening caused by the laser. The absence of laser-driven energy modulation in Fig. 10(b) also confirms that there was no residual interaction from the IFEL. To summarize, the observed data in Fig. 10 is a clear experimental confirmation of the LawsonWoodward theorem.

The dependence on laser peak electric field and polarization.-Figure 11(a) displays the average energy modulation strength as a function of the laser polarization angle and the laser electric field strength with the corresponding fitted lines to the data. The data points correspond to the averaged measured energy modulation values $\langle M\rangle$ and the vertical error bars correspond to the standard deviation of $\langle M\rangle$. The expected cosine type dependence on the laser polarization $\Delta U \propto \cos \rho$ is observed. The maximum modulation occurs at a polarization angle of $0^{\circ}$, corresponding to the laser electric field oriented in the $x z$ plane (see Fig. 1). At a polarization angle of $90^{\circ}$ the electric field is vertically polarized and as expected no significant energy modulation is observed. Figure 11(b) shows the laser electric field dependence. The linear fit of the laser peak electric field dependence is

$$
\begin{aligned}
\langle M\rangle(\mathrm{keV})= & (0.349 \pm 0.017) E_{\text {longitudinal }}(\mathrm{MV} / \mathrm{m}) \\
& -(0.35 \pm 0.25) .
\end{aligned}
$$

The observed data illustrate that there is no significant offset to the linear fit and that the laser-driven energy modulation scales linearly with the applied laser electric field that is parallel to the electron beam trajectory and confirms that $\Delta U \propto E_{0}$. The peak longitudinal electric field was not measured but was calculated from the lasercrossing angle and the transverse electric field $E_{\text {longitudinal }} \sim \alpha E_{0}$. The transverse electric field $E_{0}$ was calculated from the measured laser pulse energy and laser spot size.

The dependence on the laser-crossing angle.-As shown in Fig. 3 the energy gain $\Delta U$ is expected to follow a dependence on the laser-crossing angle that scales as $\Delta U \propto \alpha /\left(1 / \gamma^{2}+\alpha^{2}\right)$ with an expected maximum occurring at $\alpha=1 / \gamma$. Preliminary measurements that probed laser-crossing angles between 3 and $20 \mathrm{mrad}$ showed a maximum energy gain near the expected optimum crossing angle of $16 \mathrm{mrad}$, however a more complete set of measurements probing over a wider range of laser-crossing

\section{a) Laser polarization angle dependence}

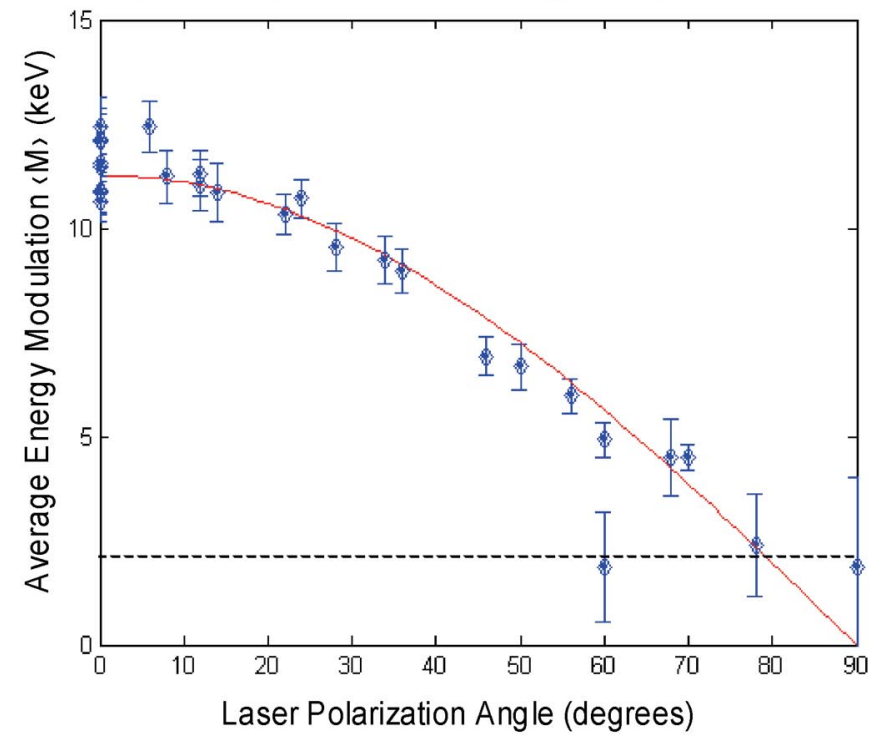

b) Laser electric field dependence

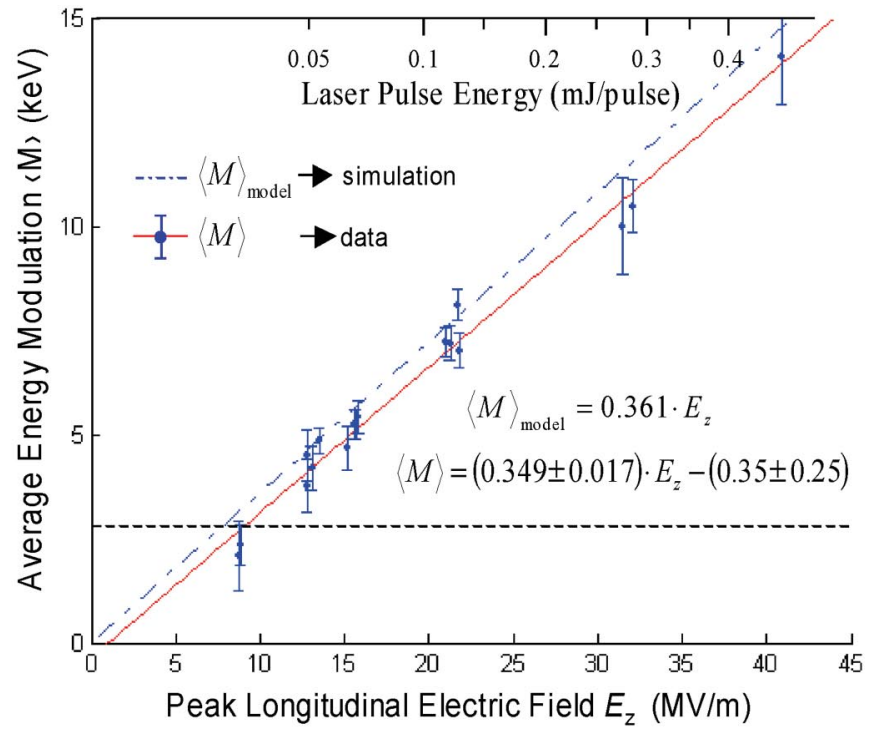

FIG. 11. (Color) (a) Dependence of the average energy modulation $\langle M\rangle$ on the laser polarization angle. The red curve is a cosine function fit to the data. (b) Dependence of the average energy modulation $\langle M\rangle$ on the laser peak electric field. The red line is a linear fit to the data. The dashed lines in both plots show the noise floor limit. 
angles will be required to quantitatively test the expected dependence on laser-crossing angle.

To summarize, the energy modulation was found to scale linearly with the laser electric field amplitude and to agree with the expected energy modulation strength. Furthermore it was seen to follow the expected cosine dependence on the polarization angle. Finally it was observed that in accordance to the Lawson-Woodward theorem energy modulation occurred only in the presence of a field-terminating boundary.

\section{FUTURE EXPERIMENTS}

In upcoming accelerator runs we plan to improve the angular beam alignment repeatability to perform detailed studies of the dependence of the energy modulation on the laser-crossing angle and to make a quantitative comparison with the expected $\Delta U \propto \alpha /\left(1 / \gamma^{2}+\alpha^{2}\right)$ dependence on laser-crossing angle. Furthermore, the thin-tape setup described in this paper is ideally suited for study of the potential effect of the optical properties of the boundary on the observed laser acceleration. Different types of boundary surfaces such as transmissive, diffusive, or absorbing thin tapes will allow for a direct experimental test on the validity of the equivalence between the inverseradiation picture and the path integral method. Finally we plan to perform a staged accelerator experiment in a similar fashion as has been carried out with staged IFELs [25] by using the IFEL as an optical buncher and the tape surface a laser accelerator in an attempt to shift the mean energy of the electron bunch. These will still be proof-ofprinciple type experiments that are aimed at studying the physics and do not pay particular attention to accelerator cell energy efficiency and energy gradient. However, considerable thought is already being given to these aspects $[26,27]$ and experiments featuring more efficient, higher impedance and higher gradient accelerator structures $[28,29]$ are in the design phase.

\section{CONCLUSIONS}

The initial proof-of-principle experiment succeeded in confirming the physics for laser-driven particle acceleration in vacuum by verifying linear dependence on the laser electric field, the expected polarization dependence, and the consistency with the Lawson-Woodward theorem. Still, there is a series of measurements with this single-boundary setup that further explores the physics of particle acceleration which are pending. Of most interest is the experimentation with different boundary types; a very simple experiment whose outcome is expected to provide a definitive confirmation of laser acceleration in vacuum as an inverse-radiation process.

\section{APPENDIX A: TRANSIENT REFLECTIVITY DURING THE LASER ABLATION}

The effectiveness of a field-terminating boundary during the presence of the laser pulse is of key importance for our experiment. Studies on ultrafast laser ablation dynamics on metal surfaces predict a few-psec survivability of the reflective gold coating in the presence of a high-fluence laser pulse. To confirm the literature findings the setup shown in Fig. 12 was constructed. The laser could be triggered manually to deliver individual pulses. To avoid changes in the laser beam profile due to changes of thermal lensing inside the laser, external attenuators were employed to control the laser pulse fluence. The laser beam was focused at low power on a clean gold-coated Kapton surface and a gated camera monitored the reflected spot. After capturing an image of the reflected spot at low power the attenuators in front of the boundary were removed and placed in front of the gated camera. Then the laser was triggered and a camera captured the reflected first high power pulse incident on the boundary. Images of the reflected laser beam at low power and the first high power laser pulse are shown in Fig. 12. As expected a second high power laser pulse on the same location of the boundary was observed to produce a
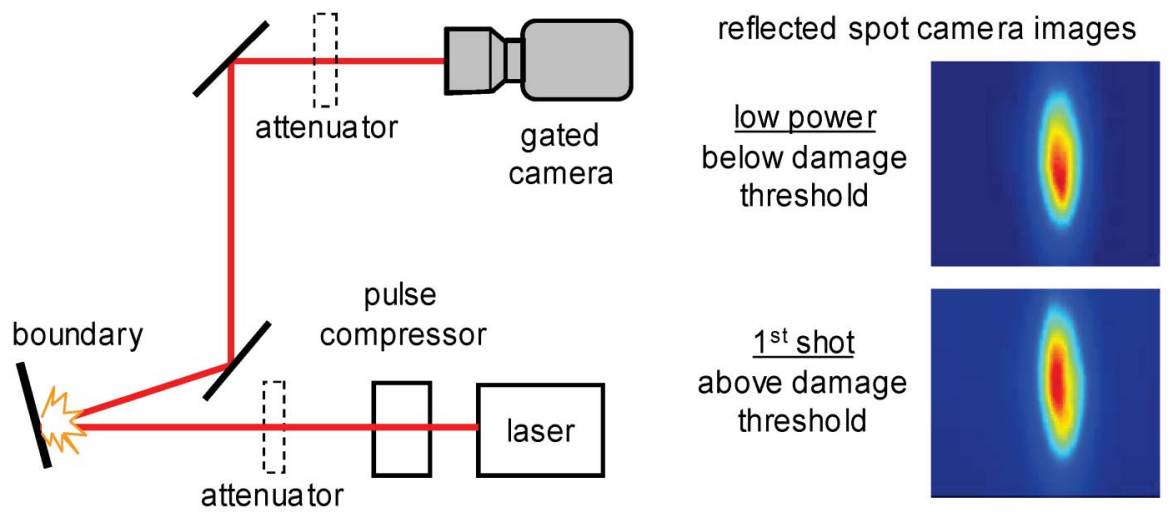

FIG. 12. (Color) The setup for the reflectance measurement from the gold-coated Kapton tape above damage threshold. The reflected spot images below damage threshold and the 1st reflected pulse above damage threshold are shown. 
TABLE II. Some representative materials and characteristics for $T_{e}=30 / 60 \mathrm{MeV}$ electrons.

\begin{tabular}{lcccccc}
\hline \hline Material & $\begin{array}{c}\text { Density } \\
\mathrm{g} / \mathrm{cm}^{3}\end{array}$ & $\begin{array}{c}\langle I\rangle^{\mathrm{a}} \\
\mathrm{eV}\end{array}$ & \multicolumn{2}{c}{$\begin{array}{c}\text { Stopping power }\left(\mathrm{MeV} \mathrm{cm}^{2} / \mathrm{g}\right) \\
\text { Radiative }\end{array}$} & $\begin{array}{c}\text { Range } \\
\text { Collisional }\end{array}$ & $\begin{array}{c}\text { Yield }^{\mathrm{b}} \\
\Sigma E_{\gamma} / T_{e}\end{array}$ \\
\hline${ }_{6} \mathrm{C}$ & $3.52^{\mathrm{c}}$ & 78 & $0.54 / 1.18$ & $1.83 / 1.89$ & $15.10 / 26.19$ & $0.12 / 0.22$ \\
${ }_{13} \mathrm{Al}$ & 2.70 & 116 & $1.00 / 2.15$ & $1.74 / 1.81$ & $14.50 / 23.56$ & $0.20 / 0.33$ \\
$\mathrm{Al}_{2} \mathrm{O}_{3}$ & 3.98 & 145 & $0.86 / 1.84$ & $1.75 / 1.81$ & $14.82 / 24.51$ & $0.18 / 0.30$ \\
${ }_{14} \mathrm{Si}$ & 2.33 & 173 & $1.11 / 2.37$ & $1.81 / 1.87$ & $13.83 / 22.310$ & $0.21 / 0.35$ \\
$\mathrm{SiO}_{2}$ & $2.42^{\mathrm{d}}$ & 139 & $0.88 / 1.90$ & $1.82 / 1.88$ & $14.30 / 23.64$ & $0.18 / 0.30$ \\
$\mathrm{PETp}^{\mathrm{e}}$ & 1.40 & 79 & $0.58 / 1.26$ & $1.94 / 2.00$ & $14.24 / 24.66$ & $0.12 / 0.22$ \\
${ }_{20} \mathrm{CaF}_{2}$ & 3.18 & 166 & $1.13 / 2.42$ & $1.75 / 1.81$ & $14.15 / 22.69$ & $0.22 / 0.36$ \\
${ }_{2} \mathrm{CrO}_{2}$ & 4.90 & 197 & $1.29 / 2.75$ & $1.84 / 1.93$ & $13.58 / 21.46$ & $0.24 / 0.36$ \\
${ }_{29} \mathrm{Cu}$ & 8.96 & 322 & $1.94 / 4.11$ & $1.56 / 1.62$ & $13.65 / 20.29$ & $0.34 / 0.50$ \\
79 & 19.3 & 790 & $3.91 / 8.23$ & $1.31 / 1.37$ & $11.69 / 15.87$ & $0.54 / 0.68$ \\
\hline \hline
\end{tabular}

${ }^{\mathrm{a}} I \equiv$ mean ionization energy is a weighted average over all levels roughly proportional to $I \sim 10 Z$ (ThomasFermi).

${ }^{\mathrm{b}}$ Yield $\equiv$ fractional energy going to bremsstrahlung with maximum transfer $E \gamma=29.75 \mathrm{MeV}$ for $T_{e}=30 \mathrm{MeV}$.

${ }^{c}$ This is diamond. Reactor grade graphite has $\rho=1.70 \mathrm{~g} / \mathrm{cm}^{3}$. Polycrystalline graphite has $\rho=2.26 \mathrm{~g} / \mathrm{cm}^{3}$.

"This is a fictitious density between amorphous, fused quartz $\left(\rho=2.20 \mathrm{~g} / \mathrm{cm}^{3}\right)$ and crystalline quartz (2.64). Borosilicate "Pyrex" and other glasses have very similar characteristics to $a$-SiO ${ }^{2}$.

${ }^{e}$ PETp is polyethylene terephthlate polyester $\left(\mathrm{C}_{10} \mathrm{H}_{8} \mathrm{O}_{4}\right)_{n}$ or "Mylar." Polyimide film $\left(\mathrm{C}_{22} \mathrm{H}_{10} \mathrm{~N}_{2} \mathrm{O}_{5}\right)_{n}$ or "Kapton" has a very similar density to Mylar so we use their average.

much weaker reflection with a significantly degraded spot quality. This confirmed the necessity to move the tape to a new location after each laser shot and brought about the idea of an audio tapelike design for the disposable boundary for the present laser-acceleration setup. A remaining question was to determine the time scale of the observed transient reflectivity behavior and to confirm its validity for a few-psec laser pulse. To this end the reflected pulse above damage threshold as a function of laser pulse duration was measured. The laser pulse duration was set by adjusting a grating-based pulse compressor at the exit of the laser. It was observed that for laser pulses with durations of $10 \mathrm{psec}$ FWHM or longer the reflected spot showed a marked decrease in its reflectivity.

\section{APPENDIX B: ELECTRON BEAM FOIL PARAMETERS}

In studying the various effects of a foil on the electron bunch characteristics a number of materials and possibilities were explored. Analytic calculations showed that the usual high-energy approximations were not valid and the electron scattering process in the foil was mostly in the plural scattering regime. Table II gives parameters for some of the options that were considered for the nominal beam energy in this experiment $(30 \mathrm{MeV})$ as well as for the follow-on experiments at $60 \mathrm{MeV}$. The Table shows that the radiative losses never dominate the collisional (excitation and ionization) except for the highest energy and $Z$ values, e.g., $\mathrm{Cu}$ has a critical energy of $25 \mathrm{MeV}$ and $\mathrm{Au}$ reaches a photon yield of only $68 \%$ at $60 \mathrm{MeV}$.

The most useful code to calculate the various effects was ELEGANT, which provided the optics and graphics needed for a complete simulation for the expected incident phase space modeling the foil and the incident electron beam.
Because the foil was at the object the observed spectrometer image was relatively insensitive to increases in emittance due to multiple Coulomb scattering. This allowed us to first concentrate on the induced energy loss and straggling before fully exploring what foils or tapes were available and preferred. We estimated that 1 mil of $\mathrm{Al}$ or its equivalent was acceptable based on matching the spectrometer resolution of a few $\mathrm{keV}$ to the standard deviation derived from the FWHM of a Gaussian centered at the most probable energy loss. This thickness was also well below the range of validity of the Moliere equation.

Typical cassette tapes have a 2-5 $\mu \mathrm{m}$ magnetic layer of material such as chromium dioxide (black) on a 6-15 $\mu \mathrm{m}$ polyester layer such as Mylar. While some of these would have been acceptable, we settled on the $1 \mu \mathrm{m} \mathrm{Au}$ on Kapton because this was highly reflective and better than our 1 mil Al benchmark. Measurements verified that there were no readily observable changes to the transverse phase space and, at most, a few percent increase in energy spread of the electron beam on inserting such tape.

Following Table II, we can make a crude estimate for comparison to Fig. 10 by taking the radiative stopping power for $1 \mu \mathrm{m}$ of gold and the collisional value for $8 \mu \mathrm{m}$ of Kapton to give a loss $\Delta U=7.5 \mathrm{keV}+$ $2.2 \mathrm{keV}=9.7 \mathrm{keV}$. Taking the spread as one third of the loss implies a standard deviation of $3.2 \mathrm{keV}$. Adding this in quadrature to the observed value of 9.4 from the $22 \mathrm{keV}$ FWHM in Fig. 10(b) implies a corresponding increase of the observed FWHM value to $23.3 \mathrm{keV}$ as observed for Fig. 10(a).

[1] Y.C. Huang, D. Zheng, W. M. Tulloch, and R. L. Byer, Appl. Phys. Lett. 68, 753 (1996). 
[2] Y.W. Chan, Phys. Lett. 35, 305 (1971).

[3] D. K. Lee, Phys. Rev. A 4, 352 (1971).

[4] J. K. Mclver and M. J. Lubin, J. Appl. Phys. 45, 1682 (1974).

[5] J. D. Lawson, IEEE Trans. Nucl. Sci. 26, 4217 (1979).

[6] R. H. Pantell and M. A. Piestrup, Appl. Phys. Lett. 32, 781 (1978).

[7] E. Esarey, P. Sprangle, and J. Krall, Phys. Rev. E 52, 5443 (1995).

[8] E. J. Bochove, G. T. Moore, and M. O. Scully, Phys. Rev. A 46, 6640 (1992).

[9] B. Hafizi, E. Esarey, and P. Sprangle, Phys. Rev. E 55, 3539 (1997).

[10] Y.C. Huang, Y. W. Lee, T. Plettner, and R. L. Byer, The Proposed Interferometric-Type Laser-Driven Particle Accelerators, AIP Conference Proc. No. 472 (AIP, New York, 1999), pp. 581-591.

[11] Y. Liu, D. Cline, and P. He, Laser Acceleration in Vacuum Using a Donut-Shaped Laser Beam, AIP Conf. Proc. No. 472 (AIP, New York, 1999), pp. 592598.

[12] T. Plettner, R. L. Byer, E. Colby, B. Cowan, C. M. S. Sears, J. E. Spencer, and R. H. Siemann, Phys. Rev. Lett. 95, 134801 (2005).

[13] A. Yariv, Optical Electonics (Saunders College Publishing, Philadelphia, 1991), 4th ed., pp. 46-49.

[14] M. O. Scully, Appl. Phys. B 51, 238 (1990).

[15] Z. Huang, G. Stupakov, and M. Zolotorev, Phys. Rev. ST Accel. Beams 7, 011302 (2004).

[16] M. Xie, in Proceedings of the Particle Accelerator Conference, Portland, OR, 2003 (IEEE, Piscataway, NJ, 2003).
[17] See for example John David Jackson, Classical Electrodynamics (John Wiley \& Sons Inc., New York, 1975), 2nd ed., Chap. 6, p. 236.

[18] T. Plettner, Report No. SLAC-TN-05-056, 2005.

[19] R. L. Byer, T. Plettner, C. D. Barnes, E. R. Colby, B. M. Cowan, R. H. Siemann, and J. E. Spencer, in Proceedings of the Particle Accelerator Conference, Chicago, IL 2001 (IEEE, Piscataway, NJ, 2001).

[20] C. M. S. Sears, E. Colby, B. Cowan, J. E. Spencer, R. H. Siemann, T. Plettner, and R. L. Byer, Phys. Rev. Lett. 95, 194801 (2005).

[21] R. L. Byer, T. Plettner, Y.C. Huang, E. Colby, R. L. Siemann, J.E. Spencer, H. Wiedemann, C. Barnes, T. I. Smith, and R.L. Swent, in Proceedings of the 1999 Particle Accelerator Conference, New York, 1999 (IEEE, Piscataway, NJ, 1999), pp. 321-324.

[22] J. K. Chen and J. E. Beraun, J. Opt. A Pure Appl. Opt. 5, 168 (2003).

[23] S. Preuss, A. Demchuck, and M Stuke, Appl. Phys. A 61, 33 (1995).

[24] Lyman G. Parrat, Probability and Experimental Errors in Science (John Wiley and Sons, Inc., New York, 1961), p. 120.

[25] W. D. Kimura et al., Phys. Rev. Lett. 86, 4041 (2001).

[26] R. H. Siemann, Phys. Rev. ST Accel. Beams 7, 061303 (2004).

[27] Y. C. Neil Na, R. H. Siemann, and R L. Byer, Phys. Rev. ST Accel. Beams 8, 031301 (2005).

[28] X. Eddie Lin, Phys. Rev. ST Accel. Beams 4, 051301 (2001).

[29] B. M. Cowan, Phys. Rev. ST Accel. Beams 6, 101301 (2003). 